\title{
In vitro antimycobacterial studies of flavonols from Bauhinia vahlii Wight and Arn
}

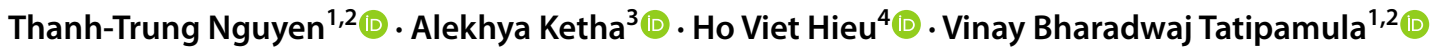

Received: 27 November 2020 / Accepted: 28 January 2021 / Published online: 16 February 2021

(c) King Abdulaziz City for Science and Technology 2021

\begin{abstract}
Mycobacterial infections and fast-growing strains are increasing globally with 8 million new cases and 1.8 million fatalities per annum worldwide. The acid-fast bacterium, Mycobacterium tuberculosis (M.t), can spread diseases like tuberculosis (Tb) and weaken the immune system. In Ayurveda, the Bauhinia genus is most valued for the treatment of tuberculosis lymphadenitis. The objective of the present study is to identify anti-tubercular compounds from the under-investigated medicinal plant $B$. vahlii Wight and Arn. using bioassay guided isolation. The antimycobacterial activity was evaluated against nonvirulent strains: Mycobacterium tuberculosis H37Ra (ATCC 25177) and Mycobacterium bovis BCG (ATCC 35743). Also, antibacterial and cytotoxicity activities were tested to identify the specificity of the isolated metabolites. Bioassay-guided isolation yielded three known flavonols, namely quercetin (1), ombuin (2), and kaempferol (3), from the methanolic extract of bark of $B$. vahlii. The results of antimycobacterial activity tests revealed that $\mathbf{2}$ showed much better mycobactericidal activity than 1 and 3 under ex vivo condition with minimum inhibitory concentration (MIC) values ranged from $0.05 \pm 0.01$ to $0.26 \pm 0.01 \mathrm{nM}$, and half-maximal inhibitory concentration $\left(\mathrm{IC}_{50}\right)$ values ranged from $2.85 \pm 0.14$ to $7.21 \pm 1.09 \mathrm{nM}$ against dormant and active forms, respectively. Also, compound 2 showed higher resistance with MIC values $>100 \mu \mathrm{g} / \mathrm{mL}$ against both Gram-positive and Gram-negative bacteria and the least cytotoxicity up to $100 \mu \mathrm{g} / \mathrm{mL}$ concentration against the tested series of cancer cell lines. The results revealed the Ayurvedic use of extracts of the Bauhinia genus for treating tuberculosis, and the key bioactive compounds were found to be flavonols (1-3). The present work provides the first evidence for the presence of antimycobacterial compounds in B. vahlii.
\end{abstract}

Keywords Antibacterial activity $\cdot$ Antimycobacterial activity $\cdot$ B. vahlii $\cdot$ Cytotoxicity $\cdot$ Flavonols

Vinay Bharadwaj Tatipamula

vinaybharadwajtatipamula@duytan.edu.vn

Thanh-Trung Nguyen

trungnt@duytan.edu.vn

Alekhya Ketha

kethaalekhya@gmail.com

Ho Viet Hieu

hoviethieu@duytan.edu.vn

1 Institute of Research and Development, Duy Tan University, Da Nang 550000, Vietnam

2 Faculty of Pharmacy, Duy Tan University, Da Nang 550000, Vietnam

3 Pharmaceutical Chemistry Department, AU College of Pharmaceutical Sciences, Andhra University, Visakhapatnam 530003, India

4 Department of Medical Microbiology and Parasitology, Faculty of Medicine, Duy Tan University, Da Nang 550000, Vietnam

\section{Introduction}

Acid-fast bacilli, Mycobacterium tuberculosis (M.t), is a huge threat to human health. The acid-fast bacterium can spread diseases like tuberculosis ( $\mathrm{Tb}$ ) and weakens the immune system (Behr et al. 1999). However, depending on the illness, $\mathrm{Tb}$ requires drug therapy of antibiotics (commonly, rifampicin and isoniazid) for at least 1-6 months (Global Tuberculosis Report 2020), and the side effects of many anti-Tb drugs are severe. Usually, the treatment also causes the occurrence of extremely drug-resistant and multidrug-resistant strains of M.t (Gagneux 2006; Paidi et al. 2017). According to the World Health Organization (WHO) Global Tb Programme Report 2020, the COVID-19 pandemic situation has had a negatively impacted on care services and detection of $\mathrm{Tb}$ cases globally and the mortality rate of $\mathrm{Tb}$ patients have also increased (Glaziou 2020). Therefore, Tb has been declared a global emergency by the 
WHO (Glaziou 2020; Rakotonirina et al. 2009). Hence, in search of alternative antimycobacterial agents, we concentrated our efforts toward screening natural sources like B. vahlii Wight and Arn. (Family: Fabaceae), against M.t strains and isolating the marker compounds.

In Ayurveda, the bark, flowers, and roots of genus Bauhinia are most valued for the treatment of scrofula, tuberculosis lymphadenitis, worm infestation, and wounds. The broad spectrum of biological activities of this genus is mainly due to the presence of flavonols, flavanones, bibenzyls, triterpenes, flavonol-glycosides, saponins, and phenanthraquinones. The antimycobacterial activity of some flavanones and bibenzyls from Bauhinia purpurea has already been reported (Boonphong et al. 2007). With this background, we have presented results of bioassay-guided isolation and characterization of flavonols from $B$. vahlii and their antimycobacterial activity in both in vitro and ex vivo conditions.

\section{Materials and methods}

\section{Plant material}

Bark of B. vahlii Wight and Arn. was collected at Seshachalam hills (Tirupati), India, in Mar 2019, and a voucher specimen (DB-SVU-2019-3478) was deposited at the Department of Botany, Sri Venkateswara University, Tirupati, India.

\section{Extraction and bioassay-guided isolation}

Dried bark (about $250 \mathrm{~g}$ ) was powdered and extracted by the maceration method (Tatipamula et al. 2020) using $90 \%$ methanol $(3 \times 500 \mathrm{~mL} \times 7$ days $)$ at $25^{\circ} \mathrm{C}$. All the extract was combined and evaporated under low pressure using rotavapor (Shimadzu Rotation evaporator QR 2005-S, Japan) to obtain crude methanol extract of bark of B. vahlii (ME, $4.5 \mathrm{~g}$ ) as a dark black solid. ME was subsequently screened and found to be effective against M. tuberculosis (M.t) H37Ra (ATCC 25177) (Table 1).

About $3.0 \mathrm{~g}$ of ME was subjected to column chromatography (sintered disc column, $600 \mathrm{~mm} \times 45 \mathrm{~mm}$; Product code: 6101067, Borosil, India) over silica gel (230-400 mesh, CAS No.: 112926-00-8, Merck) by employing a mobile phase hexane-ethyl acetate gradient $(0-100 \%)$ yielding seven fractions (F1-7). These fractions were concentrated in a vacuum and screened in an antimycobacterial assay using M.t H37Ra (ATCC 25177) strain (Singh et al. 2015; Paidi et al. 2018).

From these results, it was evident that two fractions (F4 and F6) exhibited prominent inhibitory action against M.t H37Ra, suggesting further purification to identify marker compounds. F4 $(250 \mathrm{mg})$ after purification by repeated
Table 1 Yield and primary screening of anti-tubercular activity of methanol extract (ME), fractions (F1-7), and compounds (1-3) from B. vahlii Wight and Arn

\begin{tabular}{llrrr}
\hline Sample & Yield $(\mathrm{mg})$ & \multicolumn{3}{c}{$\%$ inhibition of $M . t \mathrm{H} 37 \mathrm{Ra}(\mathrm{ATCC} 25177)^{\mathrm{a}}$} \\
\cline { 3 - 5 } & & \multicolumn{1}{c}{$10 \mu \mathrm{g} / \mathrm{mL}$} & $30 \mu \mathrm{g} / \mathrm{mL}$ & $100 \mu \mathrm{g} / \mathrm{mL}$ \\
\hline ME & 4000 & $48.63 \pm 1.20$ & $68.58 \pm 1.82$ & $81.71 \pm 3.10$ \\
F1 & 800 & $2.19 \pm 0.30$ & $2.73 \pm 0.57$ & $4.80 \pm 0.28$ \\
F2 & 450 & $0.69 \pm 0.10$ & $1.31 \pm 0.12$ & $2.01 \pm 0.79$ \\
F3 & 620 & $9.86 \pm 1.02$ & $14.77 \pm 2.60$ & $28.49 \pm 0.70$ \\
F4 & 250 & $65.07 \pm 1.57$ & $76.84 \pm 1.56$ & $90.01 \pm 3.06$ \\
F5 & 310 & $16.69 \pm 0.79$ & $20.69 \pm 1.91$ & $28.49 \pm 0.70$ \\
F6 & 200 & $56.69 \pm 2.46$ & $68.14 \pm 1.59$ & $90.96 \pm 3.72$ \\
F7 & 550 & $18.85 \pm 2.81$ & $22.13 \pm 2.19$ & $34.21 \pm 4.18$ \\
1 & 80 & $66.65 \pm 6.90$ & $80.53 \pm 5.88$ & $89.19 \pm 2.20$ \\
2 & 60 & $71.22 \pm 5.19$ & $82.19 \pm 7.23$ & $93.88 \pm 3.90$ \\
3 & 50 & $23.85 \pm 5.62$ & $29.29 \pm 4.28$ & $44.41 \pm 2.67$ \\
Rifampicin & NA & $71.02 \pm 0.24$ & $92.26 \pm 2.02$ & $96.01 \pm 0.64$ \\
\hline
\end{tabular}

${ }^{\mathrm{a}}$ Mean $\pm \mathrm{SD}(n=3) ;$ M.t: Mycobacterium tuberculosis, NA not applicable

chromatography (sintered disc column, $300 \mathrm{~mm} \times 18 \mathrm{~mm}$; Product code: 6101062, Borosil, India) and preparative thin-layer chromatography (TLC) (pre-coated glass silica plates, TLC-Silica gel $60 \mathrm{GF}_{254}$, CAS: 7631-86-9, Merck, Germany) yielded 1 (80 mg). Similarly, F6 (200 mg) after purification by repeated chromatography (sintered disc column, $300 \mathrm{~mm} \times 18 \mathrm{~mm}$; Product code: 6101062, Borosil, India) using hexane-ethyl acetate gradient (0-100\%) and preparative TLC (TLC-Silica gel $60 \mathrm{GF}_{254}$, CAS: 7631-869, Merck, Germany) yielded compounds 2 (60 $\mathrm{mg}$ ) and $\mathbf{3}$ $(50 \mathrm{mg})$. Furthermore, proton $(1 \mathrm{H})$-nuclear magnetic resonance (NMR) and Carbon-13 (13C)-NMR (Bruker Avance 400 Spectrometer, Germany), Mass (LC/MS Triple Quad Portfolio, Agilent, China) spectral analyses, melting point determination (M-560/565 Melting Point Apparatus, Buchi, Switzerland), and elemental analyses (2400 CHNS Organic Elemental Analyzer, PerkinElmer, USA) were applied for the structure elucidation of isolated compounds. The measurements of Mass and NMR spectrophotometers were made using Robust mass spectrometry application software (Sturm et al. 2008) and Bruker's topspin software (Baldisseri and Biospin 2018), respectively.

\section{In vitro and ex vivo antimycobacterial assay}

In the current study, antimycobacterial activity was evaluated against a non-virulent strain of M.t in a liquid medium in triplicate. Standard cultures of M.t H37Ra (ATCC 25177) and Mycobacterium bovis BCG (ATCC 35743) were procured from the American Type Culture Collection (ATCC, Manassas, Virginia). M.t and M. bovis were grown and 
maintained as per standard protocols (Singh et al. 2015; Paidi et al. 2018). The bark extract (ME), fractions (F17), and isolated compounds (1-3) were screened for M.t inhibitory activity at 10,30 , and $100 \mu \mathrm{g} / \mathrm{mL}$ concentrations using 2,3-bis-(2-methoxy-4-nitro-5-sulfophenyl)-2H-tetrazolium-5-carboxanilide (XTT) reduction menadione assay (XRMA) at $470 \mathrm{~nm}$ (Singh et al. 2011). The inhibition of M. bovis was estimated using nitrate reductase (NR) assay at $540 \mathrm{~nm}$ (Singh et al. 2015). Through dose-response curves, minimum inhibitory concentration (MIC), and half-maximal inhibitory concentration $\left(\mathrm{IC}_{50}\right)$ values were calculated against mycobacteria strains. Rifampicin was taken as a standard.

\section{In vitro antibacterial assay}

To determine specificity, compounds $\mathbf{1 - 3}$ at $0.1,0.3,1,3,10$, 30 , and $100 \mu \mathrm{g} / \mathrm{mL}$ concentrations were tested against bacteria strains [two Gram-positive: S. aureus (ATCC25923), B. subtilis (ATCC21332), and two Gram-negative: S. typhi (ATCC1408), E. coli (ATCC25922)] in triplicate. In a 96-well microtitre plate, $1 \% \mathrm{v} / \mathrm{v}$ LB broth and the optical density $(\mathrm{OD})$-adjusted culture $(\mathrm{OD} 620=1)$ were inoculated (Dzoyem et al. 2013; Shetty et al. 2014). Then, isolated compounds (1-3) of $2.5 \mu \mathrm{L}$ and culture $(247.5 \mu \mathrm{L})$ were added and incubated at $37^{\circ} \mathrm{C}$ for $18 \mathrm{~h}$. Wells for sterility control and growth were also included, and streptomycin was used as a standard. The absorbance was measured at $620 \mathrm{~nm}$. Through dose-response curves, $\mathrm{MIC}$ and $\mathrm{IC}_{50}$ values were calculated against strains.

\section{Cytotoxicity assay}

The breast (MDA-MB-231), cervical (HT-3), and ovarian (SKOV3) cancer cell lines were procured from the National Centre for Cell Science, Pune, India, and maintained according to the standard established procedures (Chitturi et al. 2015, 2016). In vitro potential effects of compounds (1-3) on cell death were investigated through 3-(4, 5-dimethylthiazol-2-yl)-2,5- diphenyltetrazolium bromide (MTT) assay in triplicate (Tatipamula and Vedula 2018; Tatipamula et al. 2019). Through dose-response curves, $\mathrm{IC}_{50}$ values were calculated against cancer cell lines.

Fig. 1 Chemical representation of isolated flavonols (1-3) from B. vahlii<smiles></smiles>

Quercetin (1)

\section{Results and discussion}

To identify the bioactive antimycobacterial compounds from ME, it was fractionated using column chromatography, into seven fractions, namely F1-7. They were then screened for inhibitory action against M.t H37Ra. In preliminary screening, $~ 90 \%$ inhibition was observed for only ME, F4, and F6; others showed $>28 \%$ inhibition against $M . t \mathrm{H} 37 \mathrm{Ra}$ strain (Table 1). Further purification of $\mathbf{F 4}$ and $\mathbf{F 6}$ yielded compounds 1-3. By elemental and spectral analysis, these compounds were identified as quercetin (1), ombuin (2), and kaempferol (3) (Fig. 1).

Compounds 1 and 2 obtained from F4 and F6, respectively, showed profound mycobactericidal strength, inhibiting $~ 90 \%$ of mycobacterial growth. Depending upon primary screening, active compounds $\mathbf{1}$ and $\mathbf{2}$ at a concentration range of $0.03-30 \mu \mathrm{g} / \mathrm{mL}$ were further evaluated to determine their $\mathrm{IC}_{50}$ and MIC values against dormant (12 days' incubation) and active ( 8 day's incubation) forms of $M . t$ in both in vitro (M.t and M. bovis BCG), and ex vivo (M.t) conditions using the XRMA and NR assays. The results revealed the strong antimycobacterial activity of compounds $\mathbf{1}$ and 2 (Fig. 2).

Compound 1 was found to be extremely effective in inhibiting both active and dormant forms of $M$. bovis BCG (in vitro), and M.t (ex vivo) with MIC values ranged from $3.21 \pm 0.09$ to $10.26 \pm 0.73 \mathrm{nM}$ (Fig. $2 \mathrm{e}, \mathrm{f}$ ), and $\mathrm{IC}_{50}$ values ranged from $0.13 \pm 0.01$ to $4.90 \pm 0.60 \mathrm{nM}$ (Fig. $2 \mathrm{~b}, \mathrm{c}$ ). A higher concentration of compound $\mathbf{1}$ was required for complete inhibition of both active and dormant forms of M.t (in vitro) (Fig. 2a, d). Alternatively, compound 2 showed much better mycobactericidal activity under ex vivo conditions with MIC values of $0.05 \pm 0.01$ and $0.26 \pm 0.01 \mathrm{nM}$ (Fig. 2f), and $\mathrm{IC}_{50}$ values of $2.85 \pm 0.14$ and $7.21 \pm 1.09 \mathrm{nM}$ against dormant and active forms, respectively (Fig. 2c). Compound 2 exhibited the least activity against the dormant stage of M.t (in vitro) with MIC value $\sim 300 \mathrm{nM}$ (Fig. $2 \mathrm{~d}$ ) and the $\mathrm{IC}_{50}$ value of $15.86 \pm 1.10 \mathrm{nM}$ (Fig. 2a). However, the $\mathrm{IC}_{50}$ value of compound 2 in the dormant stage of M.t (in vitro) was detected to be lesser than in its active stage. Taken together, the overall antimycobacterial activity exhibited by compounds $\mathbf{1}$ and $\mathbf{2}$ was significant, although they hold inferior potencies compared to rifampicin.<smiles>COc1cc(O)c2c(=O)c(O)c(-c3ccc(OC)c(O)c3)oc2c1</smiles>

Ombuin (2)<smiles>O=c1c(O)c(-c2ccc(O)cc2)oc2cc(O)cc(O)c12</smiles>

Kaempferol (3)

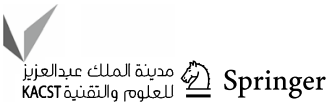



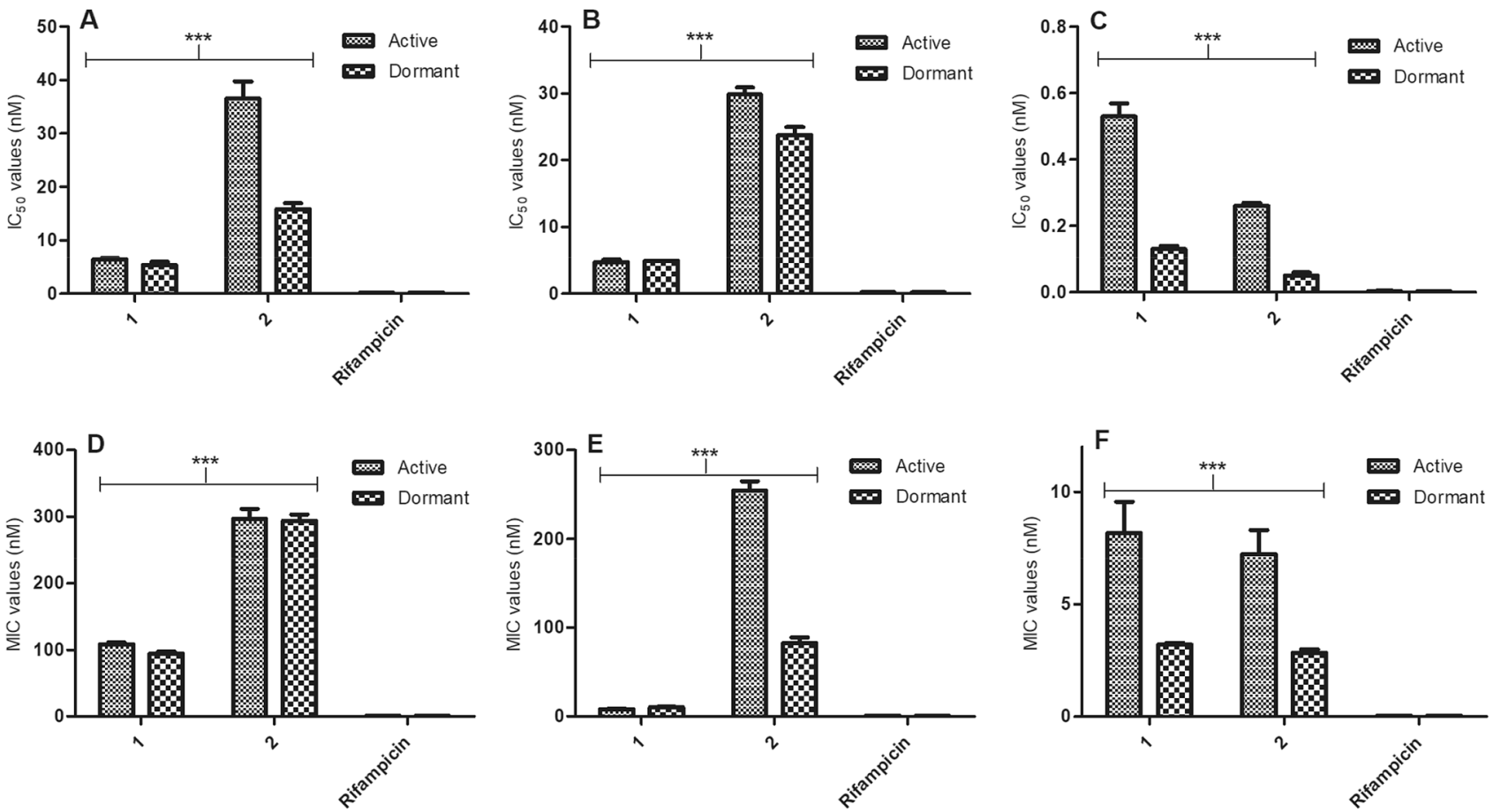

Fig. 2 Half-maximal inhibitory concentration $\left(\mathrm{IC}_{50}\right)$ values of compounds 1 and $\mathbf{2}$ against $\mathbf{a}$ in vitro $M$. tuberculosis H37Ra; $\mathbf{b}$ in vitro M. bovis BCG; c ex vivo M. tuberculosis H37Ra. Minimum inhibitory concentration (MIC) values of compounds $\mathbf{1}$ and $\mathbf{2}$ against $\mathbf{d}$ in vitro $M$. tuberculosis $\mathrm{H} 37 \mathrm{Ra}$; e in vitro $M$. bovis $\mathrm{BCG}$; F. ex vivo

To check the antimicrobial specificity of compounds 1-3, we tested them against two Gram-negative bacteria (S. typhi and $E$. coli) and two Gram-positive bacteria ( $S$. aureus and B. subtilis). Compounds $\mathbf{2}$ and $\mathbf{3}$ showed higher resistance with MIC values $>100 \mu \mathrm{g} / \mathrm{mL}$ against both Gram-positive and Gram-negative bacteria (Table 2). Moreover, the MIC values of compound 1 against tested bacterial strains ranged from $69.15 \pm 1.30$ to $151.20 \pm 5.10 \mathrm{nM}$ (Table 2). This indicates that compound $\mathbf{2}$ isolated from $\mathbf{M E}$, has greater specificity toward mycobacteria than $\mathbf{1}$.
M. tuberculosis H37Ra. *Mean $\pm \mathrm{SD}(n=3)$; Statistical analysis determined by $t$ test where $* * * p<0.0001$ is statistically significant compared to rifampicin. $\mathrm{IC}_{50}$ and $\mathrm{MIC}$ values are the lowest concentration of samples exhibiting percentage growth inhibition of $50 \%$ and $\geq 90 \%$, respectively, relative to the growth control

Furthermore, compounds 1-3 were tested against MDAMB-231, HT-3, and SKOV3 human cancer cell lines for their cytotoxicity. Fig. S1 represents the percentage of cytotoxicity and $\mathrm{IC}_{50}$ values obtained against MDA-MB-231, HT-3, and SKOV3 cancer cell lines through MTT cell proliferation assay. At $100 \mu \mathrm{g} / \mathrm{mL}$ concentration, compounds 1 and 3 exhibited 44 and $~ 53 \%$ inhibition, respectively, of MDA-MB-231 and HT-3, suggesting the biocompatible nature of these compounds. Compound 2 showed the least cytotoxicity up to $100 \mu \mathrm{g} / \mathrm{mL}$ concentration against the

Table 2 In vitro antibacterial activity of compounds (1-3) isolated from methanolic extract of bark of B. vahlii

\begin{tabular}{|c|c|c|c|c|c|c|c|c|}
\hline \multirow[t]{2}{*}{ Sample } & \multicolumn{2}{|l|}{ S. aurues $^{\mathrm{a}}$} & \multicolumn{2}{|l|}{ B. subtilis ${ }^{\mathrm{a}}$} & \multicolumn{2}{|l|}{ S. typhi ${ }^{\mathrm{a}}$} & \multicolumn{2}{|l|}{ E. coli ${ }^{\mathrm{a}}$} \\
\hline & $\mathrm{IC}_{50}$ & MIC & $\mathrm{IC}_{50}$ & MIC & $\mathrm{IC}_{50}$ & MIC & $\mathrm{IC}_{50}$ & MIC \\
\hline 1 & $41.03 \pm 2.27$ & $69.15 \pm 1.30$ & $25.81 \pm 2.18$ & $131.39 \pm 8.00$ & $80.13 \pm 4.17$ & $143.73 \pm 7.26$ & $117.22 \pm 4.35$ & $151.20 \pm 5.10$ \\
\hline 2 & $287.63 \pm 6.36$ & $290.65 \pm 10.34$ & $299.74 \pm 9.25$ & - & $207.61 \pm 15.38$ & - & $223.29 \pm 16.70$ & $267.73 \pm 9.26$ \\
\hline 3 & - & - & - & - & - & - & - & - \\
\hline Streptomycin & $0.34 \pm 0.05$ & $2.86 \pm 0.13$ & $16.86 \pm 1.13$ & $29.54 \pm 2.45$ & $8.10 \pm 0.89$ & $12.48 \pm 1.00$ & $1.17 \pm 0.13$ & $4.18 \pm 0.30$ \\
\hline Rifampicin & $19.44 \pm 1.04$ & $36.45 \pm 2.00$ & $0.30 \pm 0.03$ & $1.64 \pm 0.35$ & $0.12 \pm 0.03$ & $0.60 \pm 0.06$ & $0.81 \pm 0.09$ & $1.97 \pm 0.22$ \\
\hline
\end{tabular}

a Values are expressed as $\mathrm{nM}$ (mean $\pm \mathrm{SD}, n=3$ ); “-” indicate not active up to $100 \mu \mathrm{g} / \mathrm{mL}$ concentration; half-maximal inhibitory concentration $\left(\mathrm{IC}_{50}\right)$ and minimum inhibitory concentration (MIC) values are lowest concentration of samples exhibiting percentage growth inhibition of 50\% and $\geq 90 \%$, respectively, relative to the growth control 
tested series of cancer cell lines. The tested concentration was nearly ten times greater than the detected MIC values for M.t in ex vivo conditions. Overall, the cytotoxicity results suggest the biocompatible nature of compounds 1-3.

To conclude, the present work provides the first evidence for the presence of antimycobacterial compounds in $B$. vahlii. Hence, we report the bioassay-guided isolation of marker compounds 1-2 from the bark of $B$. vahlii, possessing significant inhibitory actions against non-virulent M.t strains (both in vitro and ex vivo). This study supports the prediction of predominant binding mode(s) of compounds $\mathbf{1}$ and $\mathbf{2}$ within M.t proteins (like DprE1) that help to recognize the ligand-protein interactions and establish a structural basis for inhibition of M.t strains.

Supplementary Information The online version contains supplementary material available at https://doi.org/10.1007/s13205-021-02672-4.

Acknowledgements The authors would like to thank AU College of Pharmaceutical Sciences, Andhra University, Visakhapatnam for providing the necessary facilities to complete the present work. The authors would also like to thank Adam F. Johnson (Institute of Research and Development, Duy Tan University, Danang, Vietnam) for the critical reading of the manuscript.

Author's contribution T-TN proposed and monitored the biological assays and co-wrote the manuscript. AK and $\mathrm{HVH}$ helped with isolation and biological evaluations. VBT conceived the study, analyzed the data, and wrote the manuscript. All authors have read and approved the manuscript.

\section{Compliance with ethical standards}

Conflict of interest The authors declare that they have no conflict of interest in the publication.

\section{References}

Baldisseri DM, Biospin B (2018) Practical aspects of fragment-based screening experiments in TopSpin. Brucker. https://www.bruke r.com/fileadmin/user_upload/8-PDF-Docs/MagneticResonance/ NMR/brochures/FBS_appsnote_T169075.pdf. Accessed on 28 Dec 2020

Behr MA, Warren SA, Salamon H et al (1999) Transmission of Mycobacterium tuberculosis from patients smear-negative for acid-fast bacilli. Lancet 353:44-449

Boonphong S, Puangsombat P, Baramee A et al (2007) Bioactive compounds from Bauhinia purpurea possessing antimalarial, antimycobacterial, antifungal, anti-inflammatory, and cytotoxic activities. J Nat Prod 70:795-801
Chitturi BR, Chanti DB, Tatipamula VB et al (2015) Isolation, structural assignment and synthesis of (S, E)-2-methyloctyl 3-(4-methoxyphenyl) propenoate from the marine soft coral Sarcophyton ehrenbergi. Nat Prod Res 29:70-76

Chitturi BR, Tatipamula VB, Dokuburra CB et al (2016) Pambanolides A-C from the South Indian soft coral Sinularia inelegans. Tetrahedron 72:1933-1940

Dzoyem JP, Guru SK, Pieme CA et al (2013) Cytotoxic and antimicrobial activity of selected Cameroonian edible plants. BMC Complement Altern Med 13:78

Gagneux S (2006) The competitive cost of antibiotic resistance in Mycobacterium tuberculosis. Science 312:1944-1946

Glaziou P (2020) Predicted impact of the COVID-19 pandemic on global tuberculosis deaths in 2020. medRxiv. https://doi. org/10.1101/2020.04.28.20079582

Global Tuberculosis Report (2020) Tuberculosis. World Health Organization Newsletters. https://www.who.int/health-topics/tuberculos is \#tab=tab_3. Accessed on 26 Dec 2020

Paidi K, Tatipamula V, Kolli M, Pedakotla V (2017) Benzohydrazide incorporated imidazo [1,2-b] pyridazine: synthesis, characterization and in vitro anti-tubercular activity. Int J Chem Sci 15:172

Paidi KR, Tatipamula VB, Kolli MK et al (2018) Synthesis of imidazo[1,2-b] pyridazine comprised piperazine, morpholine derivatives as potent antimycobacterial agents with in vivo locomotor activity. Anti Infect Agent 15:131-139

Rakotonirina E-CJ, Ravaoarisoa L, Randriatsarafara FM et al (2009) Facteurs associés à l'abandon du traitement anti-tuberculeux dans la ville d'Antananarivo. Madagascar Sante Publique (Paris) 21:139

Shetty PR, Buddana SK, Tatipamula VB et al (2014) Production of polypeptide antibiotic from Streptomyces parvulus and its antibacterial activity. Brazilian J Microbiol 45:303-312

Singh U, Akhtar S, Mishra A, Sarkar D (2011) A novel screening method based on menadione mediated rapid reduction of tetrazolium salt for testing of anti-mycobacterial agents. J Microbiol Method 84:202-207

Singh R, Nawale LU, Arkile M et al (2015) Chemical and biological metal nanoparticles as antimycobacterial agents: a comparative study. Int J Antimicrob Agent 46:183-188

Sturm M, Bertsch A, Gröpl C et al (2008) OpenMS-an open-source software framework for mass spectrometry. BMC Bioinformat 9:163

Tatipamula VB, Vedula GS (2018) In vitro anti-inflammatory and cytotoxicity studies of two mangrove associated lichens, Dirinaria consimilis and Ramalina leiodea extracts. Hygeia J drugs Med 10:16-26

Tatipamula VB, Vedula GS, Sastry AVS (2019) Antarvedisides A-B from manglicolous lichen Dirinaria consimilis (Stirton) and their pharmacological profile. Asian J Chem 31:805-812

Tatipamula VB, Annam SSP, Nguyen HT et al (2020) Sekikaic acid modulates pancreatic $\beta$-cells in streptozotocin-induced type 2 diabetic rats by inhibiting digestive enzymes. Nat Prod Res. https:// doi.org/10.1080/14786419.2020.1775226 